\title{
Evaluating Mid-infrared Spectroscopy as a New Technique for Predicting Sensory Texture Attributes of Processed Cheese
}

\author{
C. C. Fagan, ${ }^{\star 1}$ C. Everard, ${ }^{\star}$ C. P. O’Donnell, ${ }^{\star}$ G. Downey,† E. M. Sheehan, $\ddagger$ \\ C. M. Delahunty,§ and D. J. O’Callaghan\| \\ *Biosystems Engineering, UCD School of Agriculture, Food Science and Veterinary Medicine, University College Dublin, \\ Earlsfort Terrace, Dublin 2, Ireland \\ †Teagasc, Ashtown Food Research Centre, Dublin 15, Ireland \\ fDepartment of Nutritional Sciences, University College Cork, Cork, Ireland \\ §Department of Food Science, University of Otago, PO Box 56, Dunedin 9015, New Zealand \\ \|Teagasc, Moorepark Food Research Centre, Fermoy, Co. Cork, Ireland
}

\begin{abstract}
The objective of this study was to investigate the potential application of mid-infrared spectroscopy for determination of selected sensory attributes in a range of experimentally manufactured processed cheese samples. This study also evaluates mid-infrared spectroscopy against other recently proposed techniques for predicting sensory texture attributes. Processed cheeses ( $\mathrm{n}=32$ ) of varying compositions were manufactured on a pilot scale. After 2 and $4 \mathrm{wk}$ of storage at $4^{\circ} \mathrm{C}$, midinfrared spectra (640 to $4,000 \mathrm{~cm}^{-1}$ ) were recorded and samples were scored on a scale of 0 to 100 for 9 attributes using descriptive sensory analysis. Models were developed by partial least squares regression using raw and pretreated spectra. The mouth-coating and mass-forming models were improved by using a reduced spectral range (930 to $\left.1,767 \mathrm{~cm}^{-1}\right)$. The remaining attributes were most successfully modeled using a combined range (930 to $1,767 \mathrm{~cm}^{-1}$ and 2,839 to $4,000 \mathrm{~cm}^{-1}$ ). The root mean square errors of cross-validation for the models were 7.4 (firmness; range 65.3), 4.6 (rubbery; range 41.7), 7.1 (creamy; range 60.9), 5.1 (chewy; range 43.3), 5.2 (mouthcoating; range 37.4), 5.3 (fragmentable; range 51.0), 7.4 (melting; range 69.3), and 3.1 (mass-forming; range 23.6). These models had a good practical utility. Model accuracy ranged from approximate quantitative predictions to excellent predictions (range error ratio $=9.6$ ). In general, the models compared favorably with previously reported instrumental texture models and near-infrared models, although the creamy, chewy, and melting models were slightly weaker than the previously reported nearinfrared models. We concluded that mid-infrared spectroscopy could be successfully used for the nondestructive and objective assessment of processed cheese sensory quality.
\end{abstract}

Received April 12, 2006.

Accepted October 30, 2006.

${ }^{1}$ Corresponding author: colette.fagan@ucd.ie
Key words: descriptive sensory analysis, processed cheese, mid-infrared spectroscopy, chemometrics

\section{INTRODUCTION}

Over 18 million tonnes of cheese were produced worldwide in 2004, and processed cheese is an important segment of this market (Wohlfarth and Richarts, 2005). The United States, the largest producer of processed cheese (where $20 \%$ of all cheese consumed is processed cheese), produced 1,092,000 tonnes in 2003 (Wohlfarth and Richarts, 2005). In the same year, the 25 countries of the European Union produced 655,000 tonnes of processed cheese (Wohlfarth and Richarts, 2005).

Consumer preference for a food product is principally determined by its sensory characteristics. Accurate monitoring and control of sensory properties will facilitate the production of high-quality products. A number of factors determine the final quality and sensory properties of processed cheese (Carić and Kaláb, 1993). These include the processing conditions used during manufacture, the composition of the ingredients, and the proportions of those ingredients added to the blend.

Sensory profiling allows various quality attributes to be identified and their intensity determined (Brown et al., 2003). Sensory attributes are traditionally assessed by descriptive sensory evaluation using trained panelists. However, this is a time-consuming and expensive process that may lack objectivity (Blazquez et al., 2006). Although instrumental techniques such as texture profile analysis (TPA) and the 3-point bend test are available for determining the texture attributes of food products, these laboratory-based techniques are time-consuming and require the use of skilled personnel in their execution (Blazquez et al., 2006). Therefore, considerable interest exists in the development of instrumental techniques to enable more objective, faster, and less expensive assessments of cheese quality to be made, including sensory aspects (Downey et al., 2005). Such a technique would assist producers to maximize yields, increase 
throughput and efficiency, reduce labor costs, and optimize product quality, consistency, and customer satisfaction. Critical points in the manufacturing process could be monitored to ensure that the final product would meet required specifications.

Recently, Kealy (2006) examined cream cheese using TPA, one of the main instrumental techniques for texture measurement, and compared the results with those of a trained taste panel. Although a reasonably strong correlation was found between the taste panel results and TPA-derived hardness and adhesiveness parameters, the correlation for cohesiveness was not straightforward. Everard (2005) also investigated the prediction of sensory attributes of processed cheese from instrumental texture attributes derived from TPA, a compression test, and a 3-point bend test. He could predict the texture attributes of firmness, rubbery, creamy, chewy, fragmentable, and mass-forming with a good level of accuracy (Everard, 2005).

Spectroscopic analysis in combination with predictive mathematical models, developed using multivariate data analysis techniques such as partial least squares (PLS) regression, have potential use in controlling and monitoring the quality of raw materials through to the final product in food processing. In particular, infrared spectroscopy has been applied as an objective and nondestructive technique to provide a rapid and real-time analysis of both composition and quality (Downey, 1998; Lefier et al., 2000; Ozen and Mauer, 2002; Blazquez et al., 2004). Blazquez et al. (2006) modeled the sensory attributes of processed cheese using near-infrared reflectance spectroscopy and PLS regression. They found that it was possible to model a number of attributes including firmness, melting, rubbery, and creamy. Two other studies have investigated the prediction of sensory attributes in natural cheese. Downey et al. (2005) and Sørensen and Jepsen (1998) successfully demonstrated that near-infrared spectroscopy in conjunction with PLS regression can be used to predict several sensory attributes of Cheddar and Danbo cheeses, respectively. Midinfrared spectroscopy has been most widely used for determination of the fat and protein contents of cheese (Chen and Irudayaraj, 1998). Irudayaraj et al. (1999) also investigated the use of mid-infrared spectroscopy to follow texture development in Cheddar cheese during ripening. They demonstrated that springiness could be successfully correlated with a number of bands in the mid-infrared spectra. Research has shown that mid-infrared spectroscopy is a useful technique for characterizing the changes in proteins during cheese ripening (Mazerolles et al., 2001). Pillonel et al. (2003) also found that mid-infrared spectroscopy may be successfully applied to the discrimination of Emmental cheese based on geographic origin.
Table 1. Quantity of ingredients $(\mathrm{g} / \mathrm{kg})$ used in the production of experimental processed cheese samples

\begin{tabular}{lcrrr}
\hline $\begin{array}{l}\text { Sample } \\
\text { number(s) }\end{array}$ & Cheddar & Butter & Water & $\begin{array}{c}\text { Emulsifying } \\
\text { salt }\end{array}$ \\
\hline 1,10 & 838.7 & 0.0 & 161.3 & 9.7 \\
2 & 838.7 & 0.0 & 151.6 & 19.4 \\
3,11 & 838.7 & 0.0 & 141.9 & 29.0 \\
4,12 & 838.7 & 51.6 & 112.9 & 9.7 \\
5,13 & 838.7 & 51.6 & 100.0 & 19.4 \\
6,14 & 838.7 & 51.6 & 90.3 & 29.0 \\
7,15 & 838.7 & 100.0 & 61.3 & 9.7 \\
8 & 838.7 & 100.0 & 51.6 & 19.4 \\
9,16 & 838.7 & 100.0 & 41.9 & 29.0 \\
17,25 & 848.4 & 51.6 & 103.2 & 9.7 \\
18,26 & 838.7 & 51.6 & 100.0 & 19.4 \\
19,27 & 829.0 & 51.6 & 100.0 & 29.0 \\
20,28 & 751.6 & 45.2 & 203.2 & 9.7 \\
21,29 & 745.2 & 45.2 & 203.2 & 19.4 \\
22,30 & 738.7 & 45.2 & 200.0 & 25.8 \\
23,31 & 651.6 & 38.7 & 303.2 & 16.1 \\
24,32 & 645.2 & 38.7 & 303.2 & 22.6 \\
\hline
\end{tabular}

No data are currently available on the application of mid-infrared spectroscopy to determine the sensory attributes in processed cheese, or regarding evaluation of mid-infrared spectroscopy in comparison with other technologies in such an application. Therefore, the objectives of this study were to investigate the use of midinfrared spectroscopy in predicting sensory texture attributes using a range of experimentally manufactured processed cheese samples and to compare the models developed with those recently modeled using near-infrared spectra and instrumental texture attributes. These newly presented data allow for the critical evaluation of mid-infrared spectroscopy as a rapid, nondestructive technique for predicting the sensory texture attributes of processed cheese.

\section{MATERIALS AND METHODS}

\section{Processed Cheese Samples}

Thirty-two processed cheese batches were manufactured in a pilot plant at Moorepark Food Research Centre, Cork, Ireland. The ingredients and formulations are listed in Table 1 . The formulations, which were selected to provide samples with compositional ranges that extended beyond those used commercially by processed cheese manufacturers, provided samples with a wide range of sensory characteristics. The ingredients were mixed for $1 \mathrm{~min}$ in a jacketed cooker (Stephan UMM/ SK5 Universal cooker; Stephan u Söhne GmbH \& Co., Hameln, Germany). The blend was cooked at $80^{\circ} \mathrm{C}$ for 2 min by indirect steam heating. During cooking, the blend was stirred constantly using a knife at $300 \mathrm{rpm}$ and a baffle mixer at $80 \mathrm{rpm}$. The cooked blend was stored in food-grade plastic containers (225 g capacity), 
Table 2. Vocabulary of sensory attributes, their definitions, and mastication phases used to carry out the sensory analysis of processed cheese samples

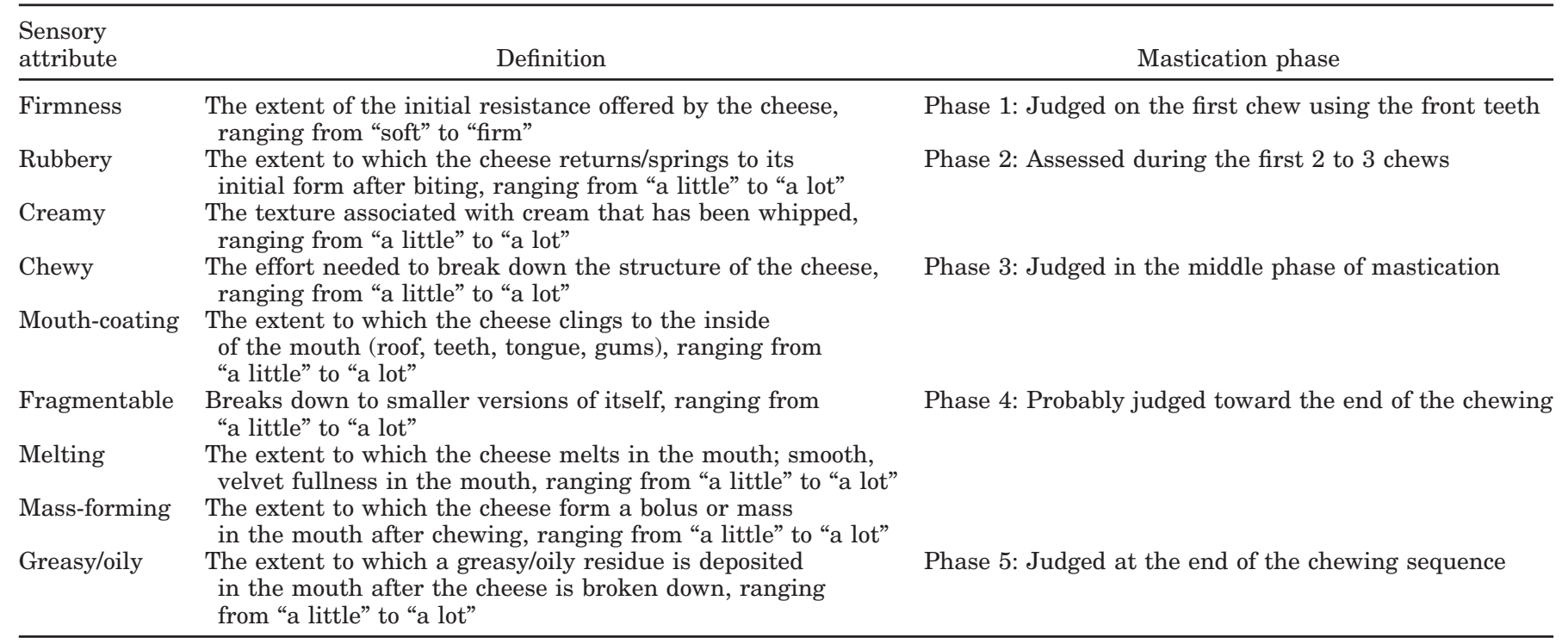

which were lidded, allowed to cool, and placed in storage at $4^{\circ} \mathrm{C}$ for $4 \mathrm{wk}$. The independent compositional variables for samples 1 to 16 were fat and emulsifying salt, and the variables for samples 17 to 32 were moisture and emulsifying salt. Descriptive sensory analysis and midinfrared spectroscopy were carried out at 2 and 4 wk postmanufacture.

\section{Sensory Analysis}

A panel of 10 assessors (9 females and 1 male), aged 35 to $55 \mathrm{yr}$ old, were selected and recruited in 1998 and 2000 according to international standards (International Organization for Standardization, 1993). The panel developed a vocabulary of 9 texture terms: firmness, rubbery, creamy, chewy, mouth-coating, fragmentable, melting, mass-forming, and greasy/oily (Table 2), which they used to assess each sample. Samples were prepared for analysis in duplicate by removing them from storage and preparing two 5-g cubes. These samples were left to equilibrate to room temperature $\left(21^{\circ} \mathrm{C}\right)$. Each equilibrated sample was presented to assessors in a glass tumbler covered with a clock glass and labeled with a randomly selected 3-digit code. Assessors were provided with deionized water and unsalted crackers to cleanse their palate between tastings. The assessors scored the samples for each attribute by marking on unstructured $100-\mathrm{mm}$ line scales labeled at both ends with extremes of each attribute. The intensity of each of the descriptive terms was recorded using the Compusense v. 4.0 sensory data acquisition software (Guelph, Ontario, Canada). At each time point, the descriptive sensory analysis took place over $2 \mathrm{~d}$. The order of tasting was balanced to account for the order of presentation and carryover effects (MacFie et al., 1989). All assessments were conducted in individual booths at the sensory laboratory at University College, Cork, which complies with international standards for the design of test rooms (International Organization for Standardization, 1988).

\section{Mid-Infrared Spectroscopy}

Mid-infrared spectra were collected over the range of 4,000 to $640 \mathrm{~cm}^{-1}$, with a resolution of $8 \mathrm{~cm}^{-1}$, using an ATI Mattson Infinity Series Fourier transform spectrophotometer (ATI Mattson, Madison, WI) controlled by WinFirst software (ATI Mattson). The sample accessory used for sample presentation was an attenuated total reflectance ZnSe crystal (Graseby Specac Ltd., Kent, UK), with an incidence angle of $45^{\circ}$ and 6 internal reflections. Sixty-four interferograms were coadded before Fourier transformation. Prior to mid-infrared analysis, samples were removed from storage and left to equilibrate to room temperature. This was confirmed prior to analysis using a digital thermocouple (Sensor-Tech Ltd., Co. Louth, Ireland). Processed cheese samples were wiped across the attenuated total reflectance crystal to ensure even and immediate contact. Triplicate spectra were captured for each sample and replicate spectra were averaged prior to data analysis.

\section{Multivariate Data Analysis}

Multivariate data analysis was carried out using The Unscrambler software (v. 8.0; Camo A/S, Oslo, Norway). 


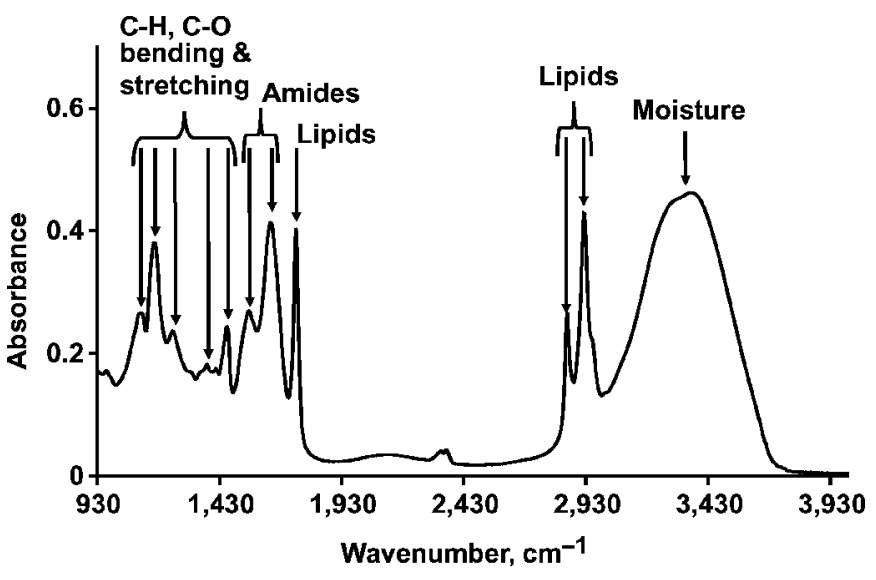

Figure 1. Typical mid-infrared spectrum of processed cheese.

Principal component analysis of the spectra was used to examine the spectral data set for any possible outliers. Models for the prediction of sensory attributes were developed using PLS regression and confirmed by crossvalidation. Prior to PLS regression, spectra were pretreated using multiplicative scatter correction (MSC), first derivative (Savitzky-Golay, 2 data points each side), second derivative (Savitzky-Golay, 4 data points each side), and each derivative plus MSC (Geladi et al., 1985). The potential of the models to predict the sensory attributes was evaluated using the root mean square error of cross-validation (RMSECV), correlation coefficient ( $\mathbf{r}$ ) and the number of PLS loadings (\#L). The range error ratio (RER) was used to determine the practical utility of the models (Williams, 1987). It was calculated by dividing the range in the reference data of a given attribute by the prediction error for that attribute. The ratio of prediction error to deviation (RPD) was calculated by dividing the standard deviation of the reference data by RMSECV.

\section{RESULTS AND DISCUSSION}

\section{Mid-Infrared Spectra}

A number of studies have assigned the main cheese constituents (fat, protein, moisture) to specific bands in the mid-infrared spectra (Chen and Irudayaraj, 1998; Chen et al., 1998; Irudayaraj and Yang, 2000; Mazerolles et al., 2001). The positions of these bands are indicated in a typical mid-infrared spectrum of processed cheese from this study (Figure 1).

The results of principal component analysis of the spectra were investigated to determine whether any influential outliers were present in the data set. An influential outlier is a sample that has both a high residual and high leverage. A high residual means that the model,
Table 3. Statistical summary of sensory attributes $(\mathrm{n}=64)$

\begin{tabular}{lcrr}
\hline $\begin{array}{l}\text { Sensory } \\
\text { attribute }\end{array}$ & Mean & Range & SD \\
\hline Firmness & 37.6 & $5.6-70.9$ & 21.9 \\
Rubbery & 23.3 & $2.9-44.6$ & 14.4 \\
Creamy & 35.7 & $10.7-70.7$ & 22.6 \\
Chewy & 24.8 & $2.8-46.1$ & 14.6 \\
Mouth-coating & 33.1 & $17.4-54.8$ & 10.0 \\
Fragmentable & 25.6 & $1.4-52.4$ & 20.0 \\
Melting & 40.1 & $13.2-82.5$ & 23.6 \\
Mass-forming & 10.5 & $1.8-25.4$ & 5.5 \\
Greasy/oily & 36.6 & $28.5-43.8$ & 3.9 \\
\hline
\end{tabular}

which nevertheless fits the other samples quite well, poorly describes the sample. Leverage measures the distance from the projected sample to the center or mean point. If a sample has a high leverage, it is exerting a stronger influence on the model than the remaining samples. According to these criteria, no outlier was found.

Previous research has recommended that prior to analysis, a portion of the mid-infrared spectra $(1,800$ to $2,700 \mathrm{~cm}^{-1}$ ) might be omitted because of its low signalto-noise ratio (Pillonel et al., 2003). This approach was used in this study, with the region 1,775 to $2,830 \mathrm{~cm}^{-1}$ having a low signal-to-noise ratio, and was therefore omitted from analysis. In a preliminary investigation of the spectra, the region 640 to $923 \mathrm{~cm}^{-1}$ was found to be of limited use in predicting sensory attributes and was also omitted. Therefore, only spectral data in the ranges of 930 to $1,767 \mathrm{~cm}^{-1}$ and 2,839 to $4,000 \mathrm{~cm}^{-1}$ were used for the multivariate data analysis.

\section{Predication of Sensory Texture Attributes by Mid-infrared Spectroscopy}

A summary of the values scored by the taste panel for each of the 9 sensory attributes is shown in Table 3 . The table highlights the high degree of variability in the data, which should support the development of robust models. Models were developed using 1) the combined spectral ranges of 930 to $1,767 \mathrm{~cm}^{-1}$ and 2,839 to $4,000 \mathrm{~cm}^{-1}$, and 2) 930 to $1,767 \mathrm{~cm}^{-1}$. The spectra were used in a number of forms: raw, MSC, first derivative, second derivative, and MSC plus each derivative step, giving 12 models for each sensory attribute. A second derivative step offered no improvement in model accuracy for any attribute; hence, those prediction results are not shown. The RMSECV, $r$, and \#L values obtained from the models developed are given in Table 4 for the combined spectral range or the 930 to $1,767 \mathrm{~cm}^{-1}$ range. These parameters allow for assessment of model strength. The preferred predictive model for an attribute (highlighted in bold in Table 4) was that which produced the lowest RMSECV 
Table 4. Summary of partial least squares prediction results for sensory attributes using mid-infrared spectra $^{1}$

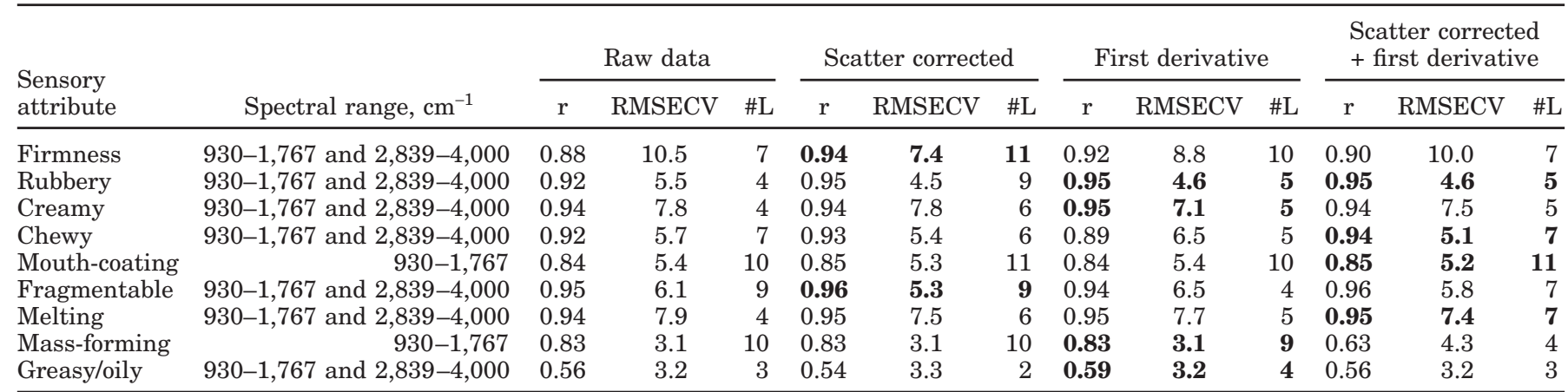

${ }^{1}$ Preferred model in bold. RMSECV = root mean square error of cross-validation; \#L = number of partial least squares loadings.

and highest $r$ values. It was also desirable for the preferred model to incorporate the lowest \#L possible.

The results showed that only 2 of the models (mouthcoating and mass-forming) were improved when the reduced spectral range (930 to $1,767 \mathrm{~cm}^{-1}$ ) was used. None of the preferred models was developed using raw spectral data (i.e., accuracy was improved by the application of a pretreatment). The firmness and fragmentable attributes were most successfully modeled using MSC spectra. The application of a first derivative step resulted in the preferred models of rubbery, creamy, mass-forming, and greasy/oily. The most accurate models for the chewy, melting, and mouth-coating attributes were achieved when the spectra were subjected to scatter correction and a first derivative.

In conjunction with the RMSECV, $r$, and \#L, the practical utility of the models can also be assessed using the RER. Models with RER of less than 3 have little practical utility; RER values of between 3 and 10 indicate limited to good practical utility; and values above 10 show that the model has a high utility value (Williams, 1987). The preferred models for predicting the firmness, rubbery, creamy, chewy, mouth-coating, fragmentable, melting, and mass-forming attributes (shown in bold in Table 4) had RMSECV values of between 3.1 and 7.4 and resulted in corresponding RER values of between 7.2 and 9.6, indicating that the models had good practical utility. Therefore, these attributes had the potential to be predicted by mid-infrared spectroscopy and multivariate data analysis. The greasy/oily attribute was not successfully modeled (RER $=4.8$ ), possibly because of the small range displayed by the samples analyzed, and will therefore not be discussed further. A graphical display of the preferred regression model for each attribute (highlighted in bold in Table 4) is shown in Figure 2A to $2 \mathrm{H}$. Figure 2 shows that there is minimal scatter in the plots, as indicated by the high $\mathrm{r}$ values ( 0.83 to 0.96 ), and that the regression lines also have slopes close to 1 (0.77 to 0.96 ) and low intercepts (1.0 to 5.9), demonstrating a good fit (Figure 2). The accuracy of each model can be evaluated using the coefficients of determination $\left(\mathrm{R}^{2}\right)$ between the predicted and measured values, as stated by Williams (2003). The models for mass-forming and mouth-coating both provided approximate quantitative predictions because their $\mathrm{R}^{2}$ lay in the range of 0.66 to 0.81 . Good predictions were achieved for the attributes firmness, rubbery, creamy, and chewy, with $\mathrm{R}^{2}$ values of between 0.82 and 0.90 . The fragmentable model was considered to be excellent, having an $\mathrm{R}^{2}$ greater than 0.91. The \#L must also be taken into account. This ranged from 5 to 11 for the selected models. The models for firmness, fragmentable, mouth-coating, and massforming incorporated a relatively high number of loadings (9 to 11), which may have implications for their robustness, because the lower $\# \mathrm{~L}$, the more robust the model.

The first 3 loadings of the models, which accounted for greater than $90 \%$ of the variation in the spectral data, are plotted in Figure 3A to 3H. Although a number of preferred models were developed using spectra pretreated with a first derivative step, interpretation of the loadings associated with these models was difficult. This was because the observed peaks and valleys did not follow the raw spectral pattern. However, second derivative steps were very helpful in spectral interpretation because in this form, band intensity and peak location were maintained with those in the raw spectral pattern. Therefore, although the second derivative step did not improve any of the prediction models, the loadings presented for rubbery, creamy, and mass-forming were obtained using second derivative spectra and those for chewy, mouth-coating, and melting were obtained using MSC second derivative spectra. The loading plots presented for firmness and fragmentable were obtained using MSC spectra. Figure 3A to $3 \mathrm{H}$ shows the relationships among the loadings used in the prediction model and the different wavenumbers. If a wavenumber had a large positive or negative loading, this meant that the 


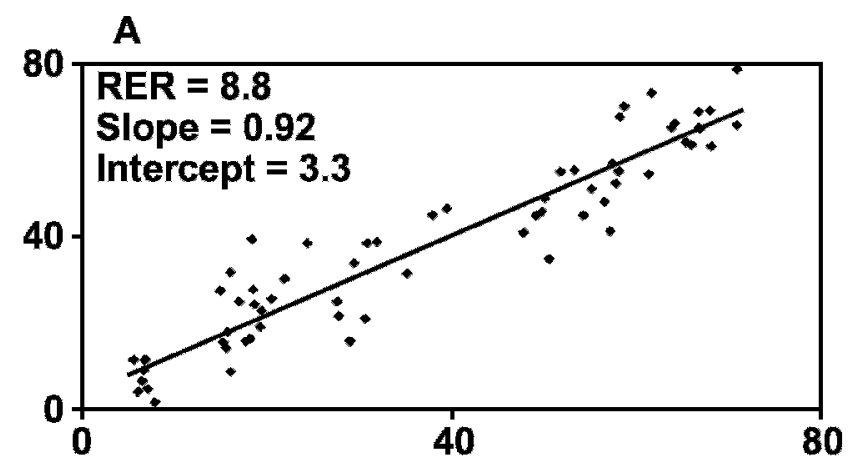

C
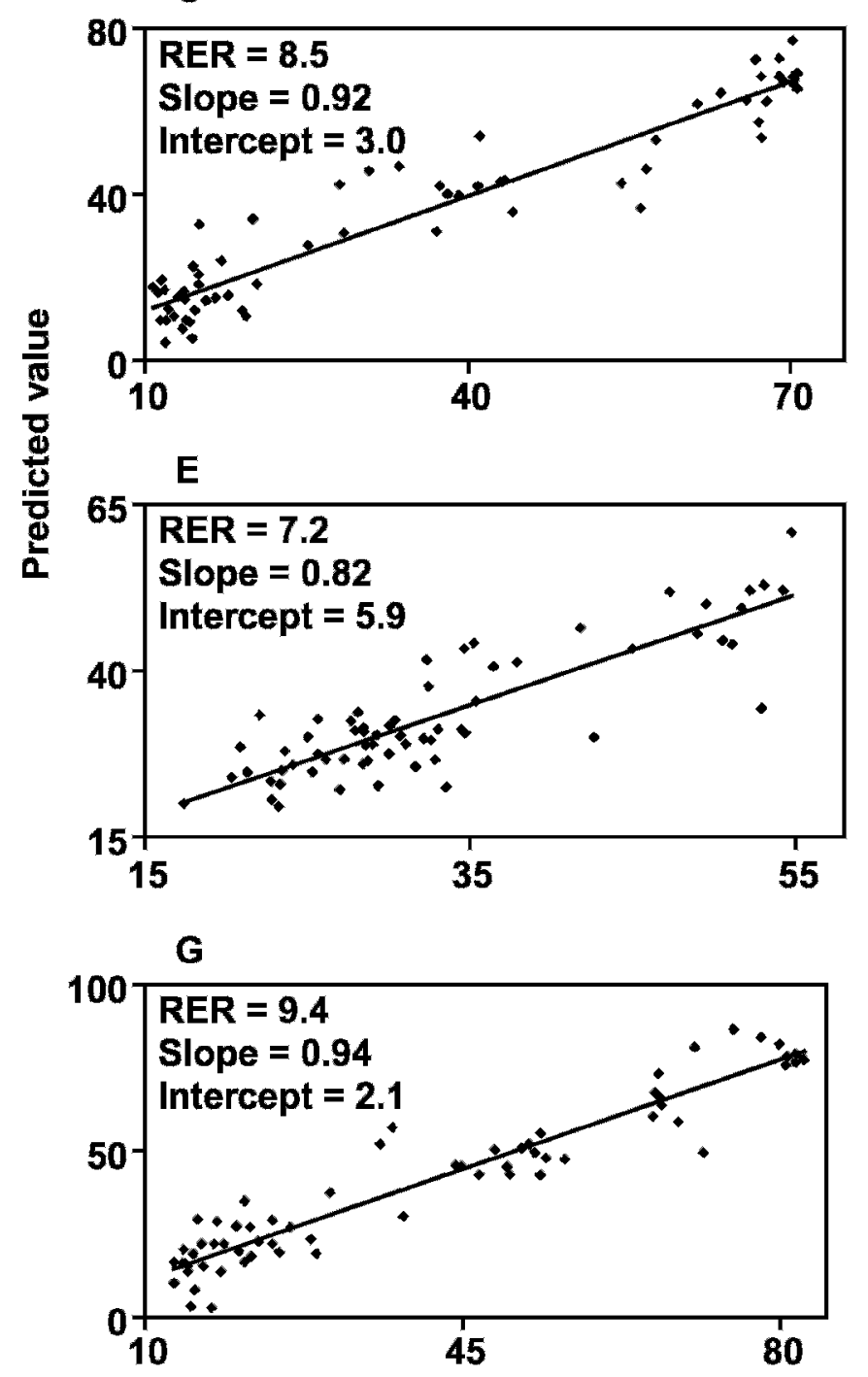

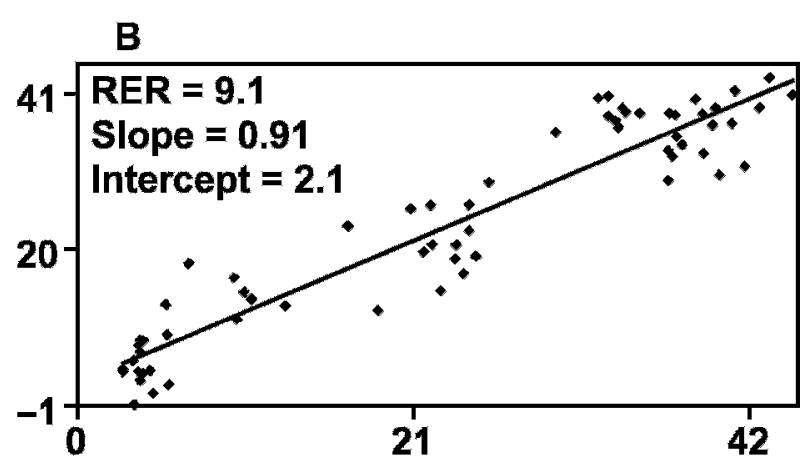

D
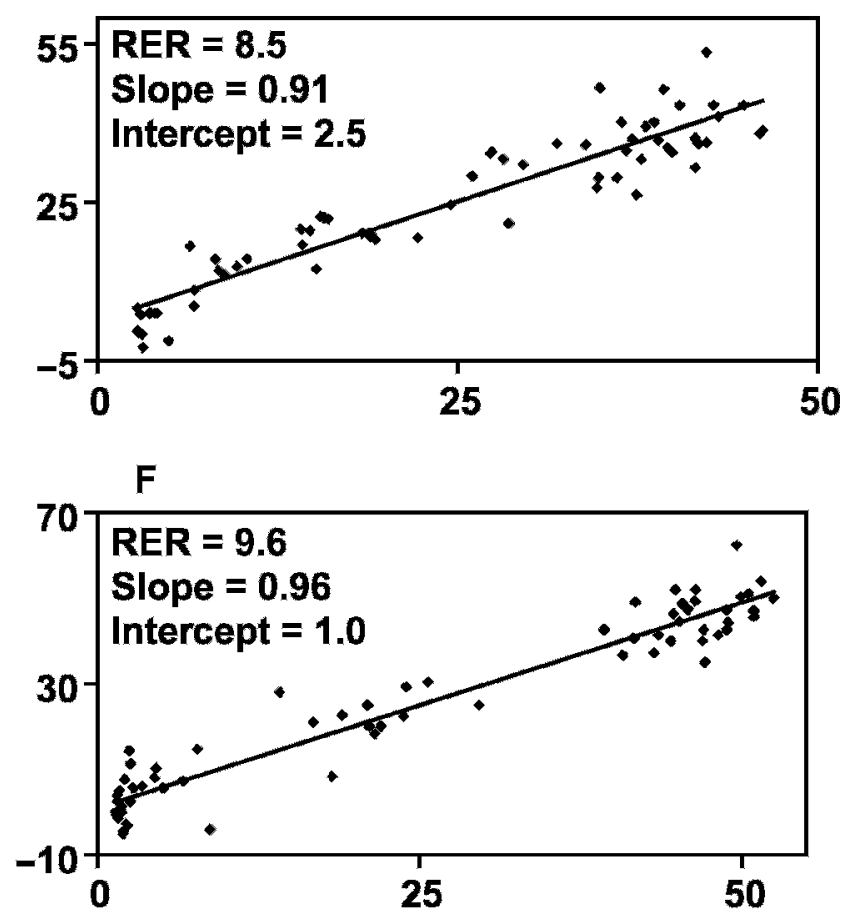

H

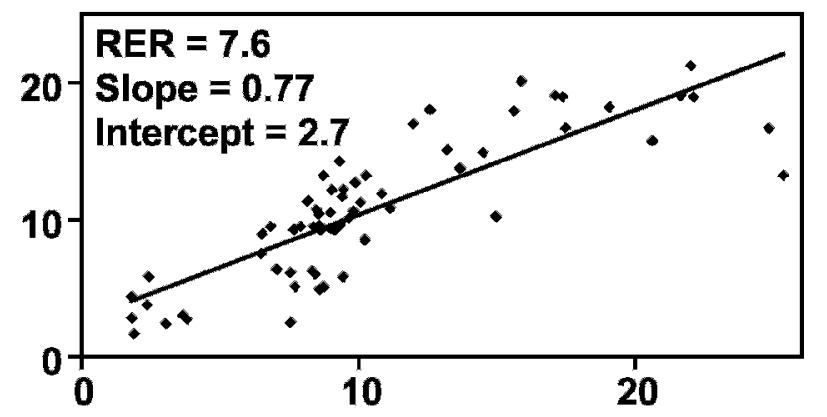

Measured value

Figure 2. Linear regression plots of actual vs. predicted sensory attributes of (A) firmness, (B) rubbery, (C) creamy, (D) chewy, (E) mouthcoating, (F) fragmentable, $(\mathrm{G})$ melting, and $(\mathrm{H})$ mass-forming. $\mathrm{RER}=$ range error ratio. 

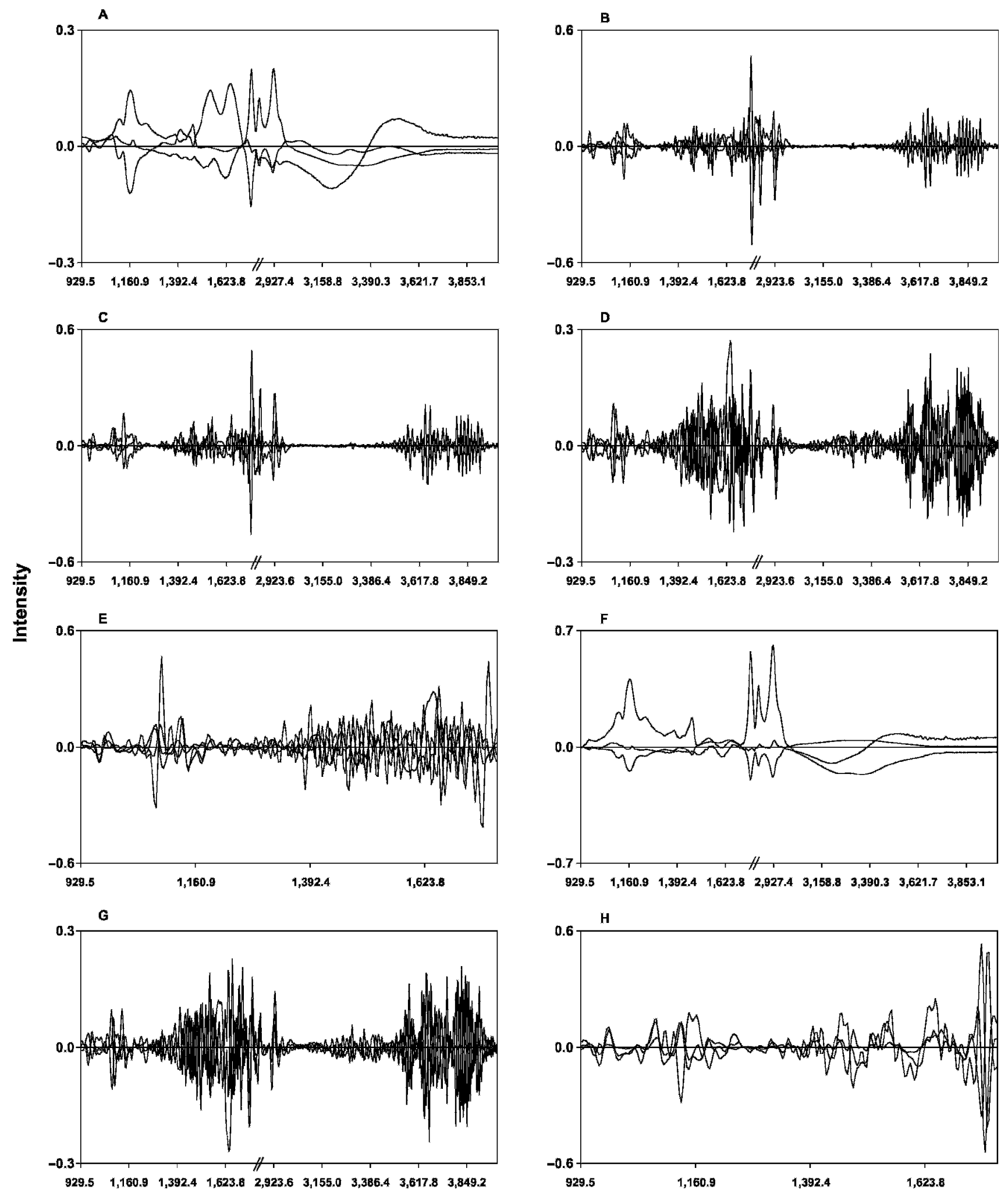

Wavenumber, $\mathrm{cm}^{-1}$

Figure 3. Loading plots for partial least squares models of the sensory attributes of (A) firmness, (B) rubbery, (C) creamy, (D) chewy, (E) mouth-coating, (F) fragmentable $(\mathrm{G})$ melting, and $(\mathrm{H})$ mass-forming. 
wavenumber was important for the attribute concerned. Therefore, they assisted in summarizing the relationship between the spectra and the predicted attribute and provided an aid to interpreting the molecular basis for predicting an attribute.

The important loadings were distributed across the full spectral range used in predicting each attribute (Figure 3). There was considerable structure present in all of the loading plots. In comparing the plots produced using similar spectral treatments, that is, MSC (Figure $3 \mathrm{~A}$ and $3 \mathrm{~F}$ ), second derivative (Figure $3 \mathrm{~B}$ and $3 \mathrm{C}$ ), and MSC plus second derivative (Figure 3D and 3G), it was apparent that differences existed in the relative importance of various regions of the spectra in predicting the different sensory attributes. For example, the region around 3,200 to $3,900 \mathrm{~cm}^{-1}$ was shown to be of greater importance in predicting the attributes of firmness (Figure 3A), chewy (Figure 3D), and melting (Figure 3G) than for the other attributes. This region of the spectra corresponded with a broad moisture absorption peak. The loadings incorporated into the firmness model explained the variation across almost the full spectral range used (Figure 3A). Peaks and valleys occurred at around $1,095,1,160$, and 1,269 to $1,396 \mathrm{~cm}^{-1}$, associated with the vibration of $\mathrm{C}-\mathrm{H}, \mathrm{C}-\mathrm{O}$ bonds of carbohydrates; $1,739,2,846$, and $2,931 \mathrm{~cm}^{-1}$, associated with lipids; and around $1,554,1,604$, and $1,646 \mathrm{~cm}^{-1}$, which are known to correspond with amides I and II. The amide I and amide II regions of the spectra were also important in predicting chewy, with peaks observed in the loading plot in the region of $1,504,1,547,1,639$, and 1,655 $\mathrm{cm}^{-1}$ (Figure 3D). The loadings for the chewy model were also found to explain variation in the moisture absorption region $\left(3,359\right.$ to $\left.3,907 \mathrm{~cm}^{-1}\right)$, the peaks associated with lipids $\left(1,739\right.$ and $\left.2,927 \mathrm{~cm}^{-1}\right)$, and the region around $1,080 \mathrm{~cm}^{-1}$. The most important regions of the spectra for predicting mouth-coating were the regions associated with the vibration of $\mathrm{C}-\mathrm{H}, \mathrm{C}-\mathrm{O}$ bonds of carbohydrates (987, 1,072 to $1,095,1,176$, and 1,334 to $1,427 \mathrm{~cm}^{-1}$ ) and lipids $\left(1,732,1,739\right.$, and $1,751 \mathrm{~cm}^{-1}$; Figure 3E). Peaks were also observed in the amide I and II region (1,542, 1,562 , and $1,655 \mathrm{~cm}^{-1}$ ). A number of major peaks were clearly identified in the fragmentable loading plot (Figure $3 \mathrm{~F})$. These were $1,110,1,169,1,242$, and 1,462 $\mathrm{cm}^{-1}$, and $1,743,2,854$, and $2,924 \mathrm{~cm}^{-1}$, which corresponded with the vibration of the $\mathrm{C}-\mathrm{H}, \mathrm{C}-\mathrm{O}$ bonds of carbohydrates and lipids, respectively. Emulsifying salts chelate calcium, which plays an important role in the 2dimensional structure of processed cheese. They also aid in the dispersion of proteins, which contributes to the emulsification of fat. In this study, the emulsifying salt used was disodium phosphate. The effect of increasing the phosphate concentration was 2-fold, namely, an increasing ability to chelate calcium and an incremental increase in the $\mathrm{pH}$ of cheese. The interaction between these 2 effects (emulsifying salt and $\mathrm{pH}$ ) will result in increased firmness of the cheese. However, this result is also dependent on the moisture content because moisture acts as a plasticizer in processed cheese and decreases the concentration of the dispersed phase, hence decreasing the firmness of the processed cheese. Greater firmness is also attributed to a higher concentration of protein, and increases in the fat and water contents weaken the protein structure, thereby decreasing the firmness of the processed cheese. This explains the importance of the fingerprint (991 to $1,400 \mathrm{~cm}^{-1}$ ), lipid, amide, and moisture-absorption regions in predicting the firmness, chewy, fragmentable, and mass-forming attributes.

The regions of the spectra that were most important in predicting the attributes of rubbery (Figure 3B) and creamy (Figure 3C) were all associated with lipids (1,739, $\left.1,743,2,846,2,858,2,916,2,919 \mathrm{~cm}^{-1}\right)$. Minor peaks were also observed in the 1,079 to $1,173,1,542$, and 3,556 to $3,907 \mathrm{~cm}^{-1}$ spectral regions, which are associated with the vibration of the $\mathrm{C}-\mathrm{H}, \mathrm{C}-\mathrm{O}$ bonds; amide II; and moisture absorption, respectively. These peaks were of particular importance in predicting the rubbery and creamy attributes, because fat in cheese has the effect of preventing the protein network of the cheese matrix from forming a tough, dense structure (Lawlor et al., 2001).

The loadings for the melting model (Figure 3G) explained variation in a number of different regions, including the amide I and II regions $\left(1,547,1,654 \mathrm{~cm}^{-1}\right)$, lipid regions $\left(1,751,2,927 \mathrm{~cm}^{-1}\right)$, and moisture-absorption region ( 3367 to $3907 \mathrm{~cm}^{-1}$ ). All factors that influence either the content or distribution of fat, or the strength of the protein network are known to influence cheese meltability (Lefevere et al., 2000). This accounts for the significance of the moisture, amide, and lipid regions of the spectra in predicting melting.

The regions of the spectra that were the most important in predicting mass-forming were found to be 1,092 and $1,130 \mathrm{~cm}^{-1}$ (C-H, C-O bond vibrations); 1,535, $1,547,1,646$, and $1,647 \mathrm{~cm}^{-1}$ (amides I and II); and 1,736, 1,743 , and $1,751 \mathrm{~cm}^{-1}$ (lipids; Figure $3 \mathrm{H}$ ). This indicates the role that the fat content and protein structure has in determining the mass-forming potential of processed cheese.

These results highlight the importance of different regions across the entire spectral range used in predicting the sensory textural attributes of processed cheese. The importance of different spectral regions in predicting sensory attributes is related to the effects of the formulation and composition on processed cheese texture. Changes in the formulation and composition of 
Table 5. Comparison of the selected mid-infrared (MIR) models to those previously developed by using near-infrared (NIR) spectroscopy (Blazquez et al., 2006), compression/texture profile analysis (TPA; Everard et al., 2006), TPA, and 3-point bend (Everard, 2005) ${ }^{1}$

\begin{tabular}{|c|c|c|c|c|c|c|c|c|c|c|c|c|c|c|c|c|c|c|c|c|}
\hline & \multicolumn{4}{|c|}{ MIR, $\mathrm{n}=64$} & \multicolumn{4}{|c|}{ NIR, $\mathrm{n}=64$} & \multicolumn{4}{|c|}{$\begin{array}{c}\text { Compression/TPA, } \\
\mathrm{n}=54\end{array}$} & \multicolumn{4}{|c|}{ TPA, $\mathrm{n}=28$} & \multicolumn{4}{|c|}{$\begin{array}{c}3 \text {-point bend, } \\
\mathrm{n}=28\end{array}$} \\
\hline & $\mathrm{R}^{2}$ & RER & RPD & $\# \mathrm{~L}$ & $\mathrm{R}^{2}$ & RER & RPD & $\# \mathrm{~L}$ & $\mathrm{R}^{2}$ & RER & RPD & $\# \mathrm{~L}$ & $\mathrm{R}^{2}$ & RER & RPD & \#L & $\mathrm{R}^{2}$ & RER & RPD & \#L \\
\hline Rubbery & 0.90 & 9.1 & 3.1 & 5 & 0.90 & 9.1 & 3.1 & 4 & 0.83 & 7.6 & 2.5 & 2 & 0.83 & 7.4 & 2.5 & 2 & 0.86 & 8.0 & 2.7 & 4 \\
\hline Creamy & 0.90 & 8.5 & 3.2 & 5 & 0.94 & 10.5 & 3.9 & 5 & 0.88 & 8.5 & 3.0 & 2 & 0.79 & 7.2 & 2.3 & 2 & 0.77 & 6.8 & 2.1 & 2 \\
\hline Chewy & 0.88 & 8.5 & 2.9 & 7 & 0.94 & 12.0 & 4.1 & 9 & 0.88 & 9.2 & 3.0 & 2 & 0.77 & 7.2 & 2.1 & 2 & 0.81 & 7.7 & 2.3 & 4 \\
\hline Mouth-coating & 0.72 & 7.2 & 1.9 & 11 & 0.79 & 8.2 & 2.2 & 10 & 0.44 & 5.1 & 1.4 & 1 & 0.01 & 3.8 & 0.9 & 3 & 0.07 & 4.3 & 1.0 & 1 \\
\hline Mass-forming & 0.69 & 7.6 & 1.8 & 9 & 0.72 & 8.2 & 1.9 & 4 & 0.34 & 4.8 & 1.2 & 1 & 0.52 & 5.2 & 1.4 & 5 & 0.42 & 4.9 & 1.3 & 1 \\
\hline Greasy/oily & 0.35 & 4.8 & 1.2 & 4 & 0.64 & 6.5 & 1.6 & 4 & 0.41 & 4.6 & 1.3 & 2 & 0.09 & 4.7 & 1.1 & 2 & 0.03 & 4.3 & 1.0 & 2 \\
\hline
\end{tabular}

${ }^{1} \mathrm{RER}=$ range error ratio; $\mathrm{RPD}=$ ratio of prediction error to deviation; $\# \mathrm{~L}=$ number of partial least squares loadings.

processed cheese directly affect its molecular structure and hence its mid-infrared spectra.

\section{Evaluation of Mid-infrared Spectroscopy vs. Other Technologies}

Recently, a small number of studies have investigated the prediction of sensory texture attributes using nearinfrared spectroscopy (Blazquez et al., 2006) and instrumental texture attributes (3-point bend test, compression test, and TPA-derived parameters; Everard, 2005; Everard et al., 2006). These models were developed using the same set of experimentally manufactured processed cheese samples as those in the current study. This provides a unique opportunity to compare the models developed using a number of different technologies. The models were evaluated using the RER, RPD, and $\mathrm{R}^{2}$ values, which are given in Table 5 . These values were calculated based on the data provided in Blazquez et al. (2006), Everard et al. (2006), and Everard (2005). Both the RER and RPD standardize the RMSECV value of the model against the range and standard deviation of the reference data, respectively. The RPD is desired to be greater that 2 for a good calibration, whereas an RPD value of less than 1.5 indicates incorrect predictions and an unusable model (Karoui et al., 2006).

Table 5 indicates that the mid-infrared models had a practical utility similar to or higher than those developed using instrumental texture data (Everard, 2005; Everard et al., 2006). Interestingly, the firmness models developed in the study by Everard (2005) using TPA and 3 -point bend test data actually produced models with a higher practical utility than did the mid-infrared models. However, one must take into account that a much smaller sample set was used in the Everard (2005) study. Models that were developed by Everard et al. (2006) using a combination of compression test and TPA data produced some results similar to the mid-infrared mod- els, as shown in Table 5 by the similar RER and RPD values.

The aim of traditional instrumental techniques for texture analysis is to replicate the actions involved in mastication. However, large deformation tests, such as TPA, involve a combination of compression, tensile, and shear forces at the microstructural level (i.e., mainly compression and shear forces in compression tests and mainly compression and tensile forces in bending tests; Van Vliet 1999). Because of the complexity of forces, large deformation tests may not measure rheological properties precisely, but may relate more accurately to the deformations involved in chewing. Firmness has been found to be strongly correlated with 2 instrumental texture parameters: the force at a given deformation (firmness $\left._{\mathrm{r}}\right)$ and fracture stress $\left(\sigma_{\mathbf{f}}\right)(\mathrm{r}>0.90 ; P<0.0001$; Everard et al., 2006). Both firmness $\mathrm{r}_{\mathrm{r}}$ and $\sigma_{\mathrm{f}}$ are related to the fracture and deformation that occur within the samples under test. Therefore, the fracture and deformation occurring in the samples during sensory analysis and instrumental texture analysis are related. This is the basis for the strong correlation between firmness and the instrumental texture attributes (firmness $s_{r}$ and $\sigma_{\mathrm{f}}$ ) and also the strength of the firmness prediction model developed by Everard et al. (2006). Everard et al. (2006) also found that the chewy attribute was strongly correlated with 3 different instrumental texture attributes, including firmness $\mathrm{r}_{\mathrm{r}}$ and $\sigma_{\mathrm{f}}(\mathrm{r}>0.87 ; P<0.0001)$. As a result, the chewy model was one of the strongest models developed using the combined compression test and TPA data. However, Everard et al. (2006) observed that not all of the sensory attributes were strongly correlated with a number of instrumental texture attributes. Three of the remaining sensory attributes (i.e., rubbery, creamy, fragmentable) were strongly correlated $(r>0.89)$ with just one instrumental texture attribute, whereas melting, mass-forming and mouth-coating displayed only weak significant correlations with the instrumental 
texture attributes (Everard et al., 2006). Kealy (2006) noted that each of the traditional laboratory-based instrumental methods for texture analysis has strengths and weaknesses in terms of the closeness of approximation to the actions of the human mouth, in the relativity or absoluteness of their measurements, and in the degree of repeatability between consecutive measurements. This indicates a limitation of the method proposed by Everard et al. (2006), because such large deformation tests cannot accurately profile the entire sensory textural experience, which would be critical in predicting the mouth-coating and mass-forming attributes. Brown et al. (2003) noted that mimicking the human sensory experience would require simulating physical changes that occur during mastication caused by saliva interactions, phase changes, and temperature changes. Therefore, one benefit of using mid-infrared spectra is that the prediction models developed are based on molecular differences among samples, which is critical in determining a range of textural attributes. Mid-infrared spectra can therefore predict sensory attributes that are either straightforward or challenging to evaluate using traditional instrumental texture analysis. This accounts for the data presented in Table 5, which show that the midinfrared models predicted the firmness and chewy attributes with an accuracy similar to that of the TPA models. However, the rubbery, mouth-coating, fragmentable, melting, and mass-forming attributes were most successfully predicted using mid-infrared spectroscopy.

The $\mathrm{R}^{2}, \mathrm{RER}$, and RPD values for the models developed by Blazquez et al. (2006) are shown in Table 5. In comparison with mid-infrared spectroscopy, near-infrared spectroscopy was particularly good at predicting creamy, chewy, and melting, with the $\mathrm{R}^{2}$ values of the nearinfrared models indicating excellent predictions as opposed to the good predictions of the mid-infrared models. The RER values for the near-infrared reflectance models indicated a high utility value (Blazquez et al., 2006), whereas the RER values obtained in this study had a good practical utility. However, the mid-infrared-derived fragmentable model had better accuracy than the nearinfrared model, with excellent and good predictions, respectively. The remaining mid-infrared models compared favorably with the results obtained by Blazquez et al. (2006) using near-infrared spectroscopy, and had RER values that differed by less than 1 when compared with those developed by Blazquez et al. (2006). The relatively high number of loadings (11) associated with the firmness model was noted previously, and this result was in contrast to the near-infrared model, which incorporated just 2. This indicates that the near-infrared model may be more robust and provide greater accuracy in the future. The remaining models incorporated a similar \#L as those reported by Blazquez et al. (2006).
It may be difficult to draw conclusions regarding the improved predictions when using one region of the electromagnetic spectrum over another. Although no information is available on the regions of the near-infrared spectra that were most important in predicting the sensory texture attributes, Blazquez et al. (2006) noted that when the near-infrared spectra were subjected to principal component analysis, samples were separated along principal component 2 on the basis of moisture content. No such clear differentiation among samples of varying moisture content was observed for the mid-infrared spectra; however, there was some separation of the samples based on the level of emulsifying salt (i.e., the level $1 \%$ group, and the level 2 and $3 \%$ groups).

The sensory attributes of other cheese products have also been predicted using infrared spectroscopy. Downey et al. (2005) and Sørensen and Jepsen (1998) modeled the sensory attributes of natural cheese using near-infrared spectroscopy. The models developed by Downey et al. (2005) using near-infrared reflectance spectra for firmness, rubbery, chewy, mouth-coating, fragmentable, melting, mass-forming, and greasy/oily attributes in Cheddar cheese had corresponding RER values of 5.1, $8.8,6.3,7.6,6.8,4.9,8.5$, and 6.6. The slopes of the regression lines in these models were also lower $(0.58$ to 0.80 ), which the authors suggested indicated the presence of significant skew in some of the prediction plots (Downey et al., 2005). The prediction accuracies of the models developed in the current work were higher than those developed by Downey et al. (2005) for Cheddar cheese. Sørensen and Jepsen (1998) applied near-infrared reflectance and transmittance to predict flavor and consistency attributes in semihard Danbo cheese. In general, they found better results using the reflectance mode rather than the transmittance mode. They obtained better results for consistency (springy, sticky, coherent, soluble, and hard) attributes ( $\mathrm{r}=0.74$ to 0.88 ; standard error of prediction $=0.64$ to $1.54 ;$ RER $=12.1$ to 7.7 ) than for flavor (cheesy, acid, sweet, and unclean) attributes $(r=0.27$ to 0.59 ; standard error of prediction $=0.58$ to 0.84 ; RER = 5.5 to 7.2; Sørensen and Jepsen, 1998).

\section{CONCLUSIONS}

Mid-infrared spectroscopy in conjunction with PLS regression was successfully used to predict several sensory attributes (firmness, rubbery, creamy, chewy, mouthcoating, fragmentable, melting, and mass-forming) in an experimentally manufactured set of processed cheese samples. The RER values (7.2 to 9.6) indicated models of good practical utility. The $R^{2}$ values indicated that the mass-forming and mouth-coating models provided approximate quantitative results $(0.69$ to 0.72$)$; the firmness, rubbery, creamy, and chewy models provided 
good prediction results ( 0.88 to 0.90$)$; and the fragmentable model produced excellent results (0.92).

Mid-infrared spectroscopy produced results similar to or better than the models previously developed using instrumental texture attributes. The mid-infrared models also compared favorably with previously reported near-infrared models, with 3 attributes modeled slightly better using near-infrared spectroscopy. These results suggest that mid-infrared spectroscopy has the potential to provide nondestructive, accurate, instantaneous measurements of processed cheese sensory texture. Therefore, these models merit further evaluation in a larger study involving commercially produced cheeses to validate these findings.

\section{ACKNOWLEDGMENT}

The authors wish to acknowledge financial support from the Irish Department of Agriculture and Food under the Food Institutional Research Measure (FIRM), supported through European Union and national funds.

\section{REFERENCES}

Blazquez, C., G. Downey, D. O'Callaghan, V. Howard, C. Delahunty, E. Sheehan, and C. P. O'Donnell. 2006. Modelling of sensory and instrumental texture parameters in processed cheese by near infrared reflectance spectroscopy. J. Dairy Res. 73:58-69.

Blazquez, C., G. Downey, C. P. O'Donnell, D. O'Callaghan, and V. Howard. 2004. Prediction of moisture, fat and inorganic salts in processed cheese by near infrared reflectance spectroscopy and multivariate data analysis. J. Near Infrared Spectrosc. 12:149158

Brown, J. A., E. A. Foegeding, C. R. Daubert, M. A. Drake, and M. Gumpertz. 2003. Relationships between rheological and sensorial properties of young cheeses. J. Dairy Sci. 86:3054-3067.

Carić, and M. Kaláb. 1993. Processed cheese products. Pages 467-505 in Cheese: Chemistry, Physics and Microbiology. 2nd ed. Vol. 2. P. F. Fox, ed. Chapman \& Hall, New York, NY.

Chen, M., and J. Irudayaraj. 1998. Sampling technique for cheese analysis by FTIR spectroscopy. J. Food Sci. 63:96-99.

Chen, M., J. Irudayaraj, D. J. McMahon, and M. X. Chen. 1998. Examination of full fat and reduced fat Cheddar cheese during ripening by Fourier transform infrared spectroscopy. J. Dairy Sci. 81:2791-2797.

Downey, G. 1998. Food and food ingredient authentication by midinfrared spectroscopy and chemometrics. TrAC Trends Anal. Chem. 17:418-424.

Downey, G., E. Sheehan, C. Delahunty, D. O'Callaghan, T. Guinee, and V. Howard. 2005. Prediction of maturity and sensory attributes of Cheddar cheese using near-infrared spectroscopy. Int. Dairy J. 15:701-709.

Everard, C. D. 2005. Determination of quality characteristics of process and Cheddar cheeses using rheological, sensory and dielectric measurement techniques. PhD Diss. University College Dublin, Dublin, Ireland.

Everard, C. D., C. P. O’Donnell, D. J. O'Callaghan, E. M. Sheehan, C. M. Delahunty, B. T. O'Kennedy, and V. Howard. 2006. Prediction of sensory textural properties from rheological analysis for process cheeses varying in emulsifying salt, protein, and moisture contents. J. Sci. Food Agric. doi:10.1002/jsfa.2756

Geladi, P., D. McDougall, and H. Martens. 1985. Linearization and scatter-correction for near-infrared reflectance spectra of meat. Appl. Spectrosc. 39:491-500.

Irudayaraj, J. S., M. Chen, and D. J. McMahon. 1999. Texture development in Cheddar cheese during ripening. Can. Agric. Eng. 41:253-258.

Irudayaraj, J. S., and H. Yang. 2000. Analysis of cheese using stepscan Fourier transform infrared photoacoustic spectroscopy. Appl. Spectrosc. 54:595-600.

International Organization for Standardization (ISO). 1988. Sensory analysis-General guidance for the design of test rooms. ISO 8589. ISO, Geneva, Switzerland.

International Organization for Standardization (ISO). 1993. Sensory analysis-General guidance for the selection, training and monitoring of assessors, Part 1. Selected assessors. ISO 8586-1. ISO, Geneva, Switzerland.

Karoui, R., A. M. Mouazen, E. Dufour, L. Pillonel, D. Picque, J. De Baerdemaeker, and J.-O. Bosset. 2006. Application of the MIR for the determination of some chemical parameters in European Emmental cheeses produced during summer. Eur. Food Res. Technol. 222:165-170.

Kealy, T. 2006. Application of liquid and solid rheological technologies to the textural characterisation of semi-solid foods. Food Res. Intern. 39:265-276.

Lawlor, B. J., C. M. Delahunty, M. G. Wilkinson, and J. Sheehan. 2001. Relationship between the sensory characteristics, neutral volatile composition and gross composition of ten cheese varieties. Lait 81:487-507.

Lefevere, I., K. Dewettinck, and A. Huyghebaert. 2000. Cheese fat as driving force in cheese flow upon melting. Milchwissenschaft 55:563-566.

Lefier, D., H. Lamprell, and G. Mazerolles. 2000. Evolution of lactococcus strains during ripening in Brie cheese using Fourier transform infrared spectroscopy. Lait 80:247-254.

MacFie, H. J., N. Bratchell, N. K. Greenhoff, and I. V. Vallis. 1989. Designs to balance the effect of order of presentation and firstorder carry-over effects in Hall tests. J. Sens. Stud. 4:129-148.

Mazerolles, G., M.-F. Devaux, G. Duboz, M.-H. Duployer, N. Mouhous Riou, and E. Dufour. 2001. Infrared and fluorescence spectroscopy for monitoring protein structure and interaction changes during cheese ripening. Lait 81:509-527.

Ozen, B. F., and L. J. Mauer. 2002. Detection of hazelnut oil adulteration using FT-IR spectroscopy. J. Agric. Fd. Chem. 50:3898-3901.

Pillonel, L., W. Luginbühl, D. Picque, E. Schaller, R. Tabacchi, and J. O. Bosset. 2003. Analytical methods for the determination of the geographic origin of Emmental cheese: Mid- and near-infrared spectroscopy. Eur. Food Res. Technol. 216:174-178.

Sørensen, L. K., and R. Jepsen. 1998. Assessment of sensory properties of cheese by near-infrared spectroscopy. Int. Dairy J. 8:863-871.

Van Vliet, T. 1999. Rheological classification of foods and instrumental techniques for their study. Pages 65-98 in Food Texture: Measurement and Perception. A. J. Rosenthal, ed. Aspen Publishers, Gaithersburg, MD.

Williams, P. C. 1987. Implementation of near-infrared technology. Pages 143-167 in Near-Infrared Technology in the Agricultural and Food Industries. 2nd ed. P. C. Williams and K. Norris, ed. American Association of Cereal Chemists, St. Paul, MI.

Williams, P. 2003. Near-infrared technology-Getting the best out of light. Page 109 in A Short Course in the Practical Implementation of Near Infrared Spectroscopy for the User. 1.1 ed. PDKProjects Inc., Nanaimo, Canada.

Wohlfarth, M., and E. Richarts. 2005. The role of processed cheese markets for the overall cheese and dairy sector. Zentral Marktund Preisberichtstelle (ZMP) GmbH, Bonn, Germany. Available: http://www.assifonte.org/english/processed_cheese/processed_ cheese_market_2005.pdf Accessed Dec. 15, 2006. 